same $\epsilon$ xactness and knowledge which he adopted in the administration of drugs. Although there could be no doubt that the best form of food for young infants was the natural supply of breast milk, circumstances at times compelled the physician to find a substitute for this, and in so doing it was necessary to remember that human milk differed widely in its quantitative composition. The physician must therefore be able, if he was to copy nature's methods, to vary the percentage of the proximate principles in the substitute food according to the requirements of the individual. The difficulty in providing a proper food for infants arose, then, from the fact that every specimen of milk used for the purpose required modifying. This modification was usually effected by a rough and empirical process, but it was very desirable to replace this by a precise and definite proceeding, so that the phvsician should be able to know the percentage composition of the food taken by the child, and should be able to vary this as might be necessary. The first essential was pure milk of good quality, of known quantitative value, and free from bacterial contamination; the second was the safe transmission of such milk from its source to the infant; and the third was the capacity to vary the composition of the food as the judgment of the physician directed, and for the latter purpose he strongly advocated the establishment of milk laboratories, where the processes of milk modification could be conducted with the same intelligence and care as ruled the conduct of the pharmacy. By certain modifications of method the same principles might be adopted in the home, but Professor Rotch held that the necessary modification could only be properly carried out on a quantitative basis, and he urged that these ends could be best attained by some such methods as he had described.

The discussion which followed this paper was interesting, the conclusions arrived at by Professor Rotch being by no means universally accepted. On all hands he was supported as to the necessity for milk being pure and uncontaminated, but the necessity for the exact modification, and especially the "laboratory" modification, was not so generally admitted. Dr. De Rothschild, of Paris, said that healthy children were readily nourished on pure cow's milk, and this by appropriate dilution could be adapted to most cases of disease. The essential thing was to be able to purchase pure milk, and if this could be done all the necessary preparation could be conducted at home under the physician's direction. Dr. Northup, of New York, supported Professor Rotch, holding that it was necessary to secure food adapted to the individual infant's requirements, and the opportunity of obtaining this in an exact fashion he regarded as a triumph of American medicine. Dr. George Carpenter held that the question of expense had to be considered, and this he feared was a practical difficulty. Dr. Eric Pritchard urged that the less interference that milk used for infant feeding was subjected to the better. The fact that mother's milk varied from time to time was, he thought, an argument against enforcing too rigid a standard in preparing a substitute. The expense of laboratory milk was a serious objection, and he held that if cream of a known strength could be obtained all necessary modifications in the milk required could be carried out at home without the aid of a laboratory. Dr. Sandwith, of Cairo, and Professor Byers, of Belfast, supported Professer Rotch.

Professor Rotch, in reply, said that his proposal was not to substitute artificial for natural feeding, but to make the former, when it was required, an exact instead of a chance proceeding. The question of expense, he thought, would not be allowed to block a reform which concerned the well-being of infant life and growth.

\section{THE TREATMENT OF INOPERABLE} CANCER.

Is opening a discussion on the treatment of inoperable cancer, Mr. Henry Morris entered into a careful review of the various remedies and methods, other than the knife, which were employed with the object of curing, ameliorating, or retarding the disease, or of preventing relapse after its removal. He came to the following conclusions, among others : (a) That the serum treatment of malignant disease is not of the slightest use in carcinoma; that not one half of the cases of spindle-celled sarcoma disappear under treatment with Coley's fluid ; that in cases of sarcoma other than spindle-celled Coley's fluid is not of value; and that the treatment by Coley's fluid has many dangers and should never be employed except in absolutely inoperable cases. (b) That Beatson's treatment is limited in its action to cases of mammary carcinoma and the local and glandular recurrences after mammary carcinoma, that only a small proportion of such cases are influenced by it, and that neither as a cure nor as a palliative can it be relied upon in any given case. (c) That in Finsen's light and in the X-rays, rodent ulcer has its most successful treatment, and that this is true not only of cases otherwise inoperable, but also of operable cases, in consequence of their excellent cosmetic results and of their effects upon non-evident foci of the disease. Nevertheless there are cases of rodent ulcer which resist these forms of treatment, and some of these cases may be successfully treated by excision and caustics. (d) That sarcoma, epithelioma and the other forms of carcinoma are best treated whenever possible by early excision; and that all forms of treatment hitherto tried in inoperable cancers of these kinds are uncertain and inconstant in their effects and untrustworthy as to the duration of the results which they produce, being in the vast majority of cases quite without palliative influence of any kind except possibly in relieving pain.

\section{LEAD POISONING.}

AN interesting discussion took place on the subject of lead poisoning, Dr. S. King Alcock, of Burslem, introducing the subject in a paper on "The advantages of periodical examination in dangerous trades." The cases to which he more particularly referred were those processes in the making of china which involved the use of lead. The points to be aimed at in periodical official examinations were the elimination of the unfit, the early detection of dangerous symptoms, and the accumulation of statistics. He thought that the economics of the question would be much altered and simplified if compensation wages were to be paid during enforced idleness after suspension. Certain workers were presented for examination who were inherently unfit to work in dangerous 